\section{NSCLC: Das Risiko der Therapiekombination}

In der Therapie des nichtkleinzelligen Lungenkarzinoms (NSCLC) werden immer häufiger gleich mehrere Wachstumsfaktorrezeptoren inhibiert. Was das für die Sicherheit der Behandlung bedeutet, wurde in einer Metaanalyse aus China untersucht.

W achstumsfaktoren wie VEGF („vascular endothelial growth factor") und EGF („epidermal growth factor") und ihre Rezeptoren sind essentiell für das Tumorwachstum. Es erscheint daher nur logisch, im Rahmen einer Krebstherapie gleich mehrere dieser einschlägigen Signalwege zu blockieren. Die Wirksamkeit der zeitgleichen Inhibition von VEGF und EGFR beim fortgeschrittenen NSCLC ist gut untersucht, doch nur in wenigen Studien konzentrierte man sich auf die Risiken dieser Kombinationstherapie.

In die vorliegende Metaanalyse schlossen chinesische Kollegen 15 randomisierte klinische Studien mit 6.919 Patienten ein, in denen das Sicherheitsprofil der kombinierten Inhibition von EGFR und VEGF beim fortgeschrittenen NSCLC mit Kontrollgruppen verglichen wurde. Die Kontrollen hatten entweder Placebo, eine einfache Hemmung von nur einem der beiden Signalwege, eine Chemotherapie oder eine Kombination aus Chemotherapie und einem Inhibitor erhalten. Endpunkte der Metaanalyse waren Behandlungsabbrüche, behandlungsbedingte Todesfälle sowie Nebenwirkungen.

3 von 4 paarweisen Vergleichen ergaben, dass unter der Kombinationstherapie mehr Patienten die Behandlung aufgrund unerwünschter Ereignisse abbrachen. Die Odds Ratio (OR) der Kombinationstherapie im Vergleich mit den verschiedenen Kontrollgruppen lag zwischen 1,97 und 2,29. Die Behandlung mit einer kombinierten Inhibitionstherapie war mit verschiedenen Nebenwirkungen vom Schweregrad 3 oder 4 assoziiert. Darunter waren Hautausschläge, Diarrhö und Bluthochdruck. Auch gab es eine signifikant höhere Inzidenz von behandlungsbedingten Todesfällen unter der Kombinationstherapie im Vergleich zur einfachen EGFR-Inhibition vor allem dann, wenn der gegen VEGFR und EGFR wirkende Inhibitor Vandetanib genutzt wurde (OR 1,97).

Fazit: Eine kombinierte Inhibitionstherapie von EGFR und VEGF bei Patienten mit fortgeschrittenem NSCLC war mit einer erhöhten Toxizität verbunden. Diese war zum Teil so stark, dass sie sowohl die Compliance empfindlich reduzierte als auch die Lebensqualität der Patienten deutlich beeinträchtigte.

Christina Berndt

Ma W et al. Safety profile of combined therapy inhibiting EFGR and VEGF pathways in patient with advanced non-small-cell lung cancer: $A$ meta-analysis of 15 phase II/III randomized trials. Int J Cancer. 2015;137(2):409-19.

\title{
Hemmung der MET-Rezeptortyrosinkinase enttäuscht beim NSCLC
}

In einer Phase-II-Studie hatte die Zugabe des MET-Rezeptortyrosinkinaseinhibitors Tivantinib zu Erlotinib beim fortgeschrittenen, nichtkleinzelligen Lungenkarzinom (NSCLC) einen Trend zur Verbesserung des Überlebens gezeigt. In einer Phase-III-Studie konnte das nicht bestätigt werden.

\footnotetext{
n der Studie nahmen 1.048 PatienA ten mit fortgeschrittenem, nicht plattenepithelialem NSCLC teil, die 1 oder 2 systemische Therapien einschließlich eines Platinsalzes erhalten hatten. Randomisiert bekamen sie entweder Erlotinib (150 mg oral 1-mal täglich) plus Tivantinib $(360 \mathrm{mg} / \mathrm{Tag}$ oral 2-mal täglich; $\mathrm{E}+\mathrm{T})$ oder Erlotinib in derselben Dosierung plus Placebo $(\mathrm{E}+\mathrm{P})$ bis zum Krankheitsprogress. Tumorproben wurden auf den EGFR- und KRASMutationsstatus sowie auf die Expression und Genamplifikation des METGens hin untersucht. Primärer Endpunkt war das Gesamtüberleben (OS). Nach der Interimsanalyse erschien ein signifikanter Vorteil der Kombination
}

unwahrscheinlich und die Studie wurde abgebrochen. Das OS betrug im Median in der Gruppe E+ T 8,5, in der Kontrollgruppe 7,8 Monate und wurde damit durch den MET-Rezeptorinhibitor nicht verbessert (Hazard Ratio [HR] 0,98, $95 \%$-Konfidenzintervall [95\%-KI] 0,841,$15 ; p=0,81$, auch wenn das PFS im $\mathrm{E}+\mathrm{T}$-Arm höher war als im E+P-Arm (median 3,6 vs. 1,9 Monate; HR 0,74, $95 \%$-KI 0,62-0,89; $\mathrm{p}<0,001)$.

Die exploratorische Subgruppenanalyse legte nahe, dass Patienten mit hoher MET-Expression (in der Studie $\mathrm{n}=211$ ) doch von der Kombination $\mathrm{E}+\mathrm{T}$ profitieren könnten (medianes OS: 9,3 vs. 5,9 Monate; HR 0,70, 95 \%-KI 0,49-1,01). Für die sehr kleine Subgruppe von Patienten mit Genamplifikationen konnte kein Trend zu einem Überlebensvorteil belegt werden. Die häufigsten Nebenwirkungen waren Hautausschläge $(\mathrm{E}+\mathrm{T}: 33,1$ vs. E+P: $37,3 \%$ ), Diarrhö (34,6 vs. $41,0 \%$ ), Asthenie oder Fatigue (43,5 vs. $38,1 \%$ ) und Neutropenie (Grad 3/4: 8,5 vs. 0,8\%).

Fazit: $\mathrm{E}+\mathrm{T}$ wird ähnlich gut vertragen wie $\mathrm{E}+\mathrm{P}$. Ein leicht verbessertes PFS unter der Kombination übersetzt sich aber nicht in einen Überlebensvorteil der Gesamtpopulation. Ob Patienten mit hoher MET-Expression profitieren und diese damit ein prädiktiver Marker für die Therapieentscheidung sein könnte, müssen weitere Studien zeigen. Friederike Klein

Scagliotti G et al. Phase III Multinational, Randomized, Double-Blind, Placebo-Controlled Study of Tivantinib (ARQ 197) Plus Erlotinib Versus Erlotinib Alone in Previously Treated Patients With Locally Advanced or Metastatic Nonsquamous Non-Small-Cell Lung Cancer. J Clin Oncol. 2015;33(24):2667-74. 